\title{
Modeling Early Postnatal Brain Growth and Development with CT: Changes in the Brain Radiodensity Histogram from Birth to 2 Years
}

\author{
(D).A. Cauley, (D). Hu, DJ. Och, (DP.J. Yorks, and DS.W. Fielden
}

\begin{abstract}
BACKGROUND AND PURPOSE: The majority of brain growth and development occur in the first 2 years of life. This study investigated these changes by analysis of the brain radiodensity histogram of head CT scans from the clinical population, $0-2$ years of age.

MATERIALS AND METHODS: One hundred twenty consecutive head CTs with normal findings meeting the inclusion criteria from children from birth to 2 years were retrospectively identified from 3 different CT scan platforms. Histogram analysis was performed on brain-extracted images, and histogram mean, mode, full width at half maximum, skewness, kurtosis, and SD were correlated with subject age. The effects of scan platform were investigated. Normative curves were fitted by polynomial regression analysis.

RESULTS: Average total brain volume was $360 \mathrm{~cm}^{3}$ at birth, $948 \mathrm{~cm}^{3}$ at 1 year, and $1072 \mathrm{~cm}^{3}$ at 2 years. Total brain tissue density showed an $11 \%$ increase in mean density at 1 year and $19 \%$ at 2 years. Brain radiodensity histogram skewness was positive at birth, declining logarithmically in the first 200 days of life. The histogram kurtosis also decreased in the first 200 days to approach a normal distribution. Direct segmentation of CT images showed that changes in brain radiodensity histogram skewness correlated with, and can be explained by, a relative increase in gray matter volume and an increase in gray and white matter tissue density that occurs during this period of brain maturation.
\end{abstract}

CONCLUSIONS: Normative metrics of the brain radiodensity histogram derived from routine clinical head CT images can be used to develop a model of normal brain development.

ABBREVIATIONS: FWHM = full width at half maximum; ID = identification; LOOCV = leave-one-out cross-validation; RMSE $=$ root-mean-square error; $R^{2}=$ coefficient of determination

$T^{\prime}$ he availability and speed of CT imaging, together with radiation dose reduction strategies, serve to maintain the role of CT in pediatric clinical care. CT is particularly amenable to quantitative measures because the Hounsfield unit, reported by the scanner, is directly related to a physical property of tissue-tissue radiodensity. The differences in tissue radiodensity between gray and white matter enable segmentation of these tissues and characterization of the products of segmentation. Segmentation per-

Received October 2, 2017; accepted after revision December 11.

From the Department of Radiology (K.A.C., S.W.F.), Biomedical \& Translational Informatics (Y.H.), and Department of Medical \& Health Physics (J.O., P.J.Y.), Geisinger Health System, Danville, Pennsylvania; and Department of Imaging Science \& Innovation (S.W.F.), Geisinger Health System, Lewisburg, Pennsylvania.

Previously presented as a scientific paper at: Annual Meeting of The Eastern Neuroradiological Society, August 24-27, 2017; Toronto, Ontario, Canada.

Please address correspondence to: Keith A. Cauley, MD, PhD, Department of Radiology, Div Neuroradiology, Geisinger Medical Center, Danville, PA 17822; e-mail: keithcauley@hotmail.com

三 Indicates article with supplemental on-line appendix and table.

Indicates article with supplemental on-line photos.

http://dx.doi.org/10.3174/ajnr.A5559 mits quantitative analysis of volumes and densities of tissue types, with the potential to further the diagnostic power of the CT scan. The risks of radiation exposure preclude prospective scanning of healthy children, however, and little research has been directed at quantitative measures of brain development using CT.

Retrospective analysis of imaging data from the clinical archive provides a data base from which to develop a model of normal brain development using CT. We used a total brain radiodensity histogram method to investigate brain tissue changes as a function of subject age. This total brain histogram approach has the advantage of being objective and reproducible, is amenable to automation, and enables the development of a model of early brain maturation. Such a model could serve as a normative baseline for quantitative assessment of normal and abnormal brain development.

\section{MATERIALS AND METHODS \\ Study Cohort}

All children 2 years of age and younger presenting for evaluation by a CT imaging system at Geisinger Health System from May 
2010 to May 2016 were retrospectively reviewed after approval by the institutional review board. The study cohort included patients from neonate to 2 years of age with no known neurologic, neurocognitive, or developmental deficits, who had normal-appearing head CT findings. All head CT studies with normal findings were additionally reviewed and confirmed by a neuroradiologist with a Certificate of Added Qualification with 6 years of attending pediatric neuroradiology experience (K.A.C.). Patients who had any focal abnormal findings on the head CT study or any subsequent brain MR imaging with any abnormal focal intensity on FLAIR or other sequences, cysts, or other nonspecific structural appearances were excluded. Patients with metal artifacts or motion-degraded or non-diagnostic-quality imaging were also excluded.

A thorough chart review was performed (K.A.C.) to identify any disease history. Most cases were of minor trauma without traumatic findings, isolated seizure, apnea, and tremor. Other cases included scalp nevus and isolated facial and neck lesions without associated syndromes or intracranial extension. Patients with any systemic or noncentral nervous system diseases (eg, diseases of the renal, gastrointestinal, cardiac systems), cancers, history of prematurity, phakomatoses, genetic or syndromic conditions, epilepsy, fracture, hemorrhage, vascular lesions (aneurysm, AVM, fistula, or steno-occlusive disease of the CNS or elsewhere), acute or recent infection and fever of unknown origin, chronic medical therapy, or endocrine symptoms/laboratory abnormal results were excluded.

One hundred twenty healthy subjects from birth to 2 years (60 males and 60 females) met the inclusion criteria and were included in the study. Cases were drawn from 3 different scan platforms and were analyzed separately. The resulting cohort consisted of 45 cases ( 21 female) scanned on scanner 1 (described below), 51 cases (29 female) scanned on scanner 2, and 24 cases (12 female) scanned on scanner 3.

\section{Imaging Methods}

To assess the variation in image histogram metrics due scan platform, we collected images from 3 different scan platforms within our health care system.

- Scanner 1 serves a community medical center, is a Brilliance 64 (Philips Healthcare, Best, the Netherlands) with helical acquisition and pediatric head CT protocol with $120 \mathrm{kV}$ (peak) and fixed $192 \mathrm{~mA}$ (rotation time $=.781$ seconds, pitch $=0.64$, effective milliampere-second $=234.3$ ).

- Scanner 2 primarily serves the emergency department of a level 1 trauma center, is a LightSpeed VCT (GE Healthcare, Milwaukee, Wisconsin) with an axial acquisition and pediatric head CT protocol with $120 \mathrm{kVp}$ and modulated milliampere, minimum 20 and maximum $75 \mathrm{~mA}$ (rotation time $=0.5$ seconds).

- Scanner 3 serves a second community medical center, is a Discovery 690 (GE Healthcare) with axial acquisition and pediatric head CT protocol with $120 \mathrm{kVp}$ and fixed $240 \mathrm{~mA}$ (rotation time $=0.5$ seconds).

Each scanner undergoes a daily quality assurance procedure, which assesses the radiodensity of water. This value must be within allowable limits, generally $0 \pm 5 \mathrm{HU}$. Drift or trending is rarely observed. In addition, scanners undergo an annual inspec- tion by a medical physicist using the American College of Radiology phantom. Acceptable ranges of Hounsfield units for clinical scanners are broad ( $-7-7$ for water, $110-135$ for acrylic). ${ }^{1}$ This testing is extended to all kilovolt(peak)s used by the scanner. Additionally, service engineers routinely test the calibration at preventive maintenance.

\section{Image Processing and Analysis}

Images were thresholded, brain-extracted, and segmented in FSL (www.fmrib.ox.ac.uk/fsl), ${ }^{2-4}$ as described in Cauley et al. ${ }^{5}$ In brief, CSF was eliminated by setting a threshold minimum at $10 \mathrm{HU}$. The upper threshold value was set at $50 \mathrm{HU}$ based on the prebrain-extracted histogram and served to eliminate skull. The FSL Brain Extraction Tool (http://fsl.fmrib.ox.ac.uk/fsl/fslwiki/BET) standard brain extraction was used with a fractional intensity threshold of 0.01 . All cases were carefully reviewed for integrity of brain extraction. No cases were eliminated on the basis of technical failure of the brain extraction protocol, and no manual masking was necessary. Brain volumes were computed in FSL from brain-extracted images.

Histograms and histogram analysis were performed in Matlab (Release 2009b; MathWorks, Natick, Massachusetts). The histogram of a normal brain follows the general appearance of the normal distribution. Matlab functions skewness and kurtosis were used to assess how far from this distribution each imaging dataset lay. In Matlab, the skewness of a distribution is defined as $s=E(\mathrm{x}-\mu)^{3} / \sigma^{3}$, where $\mu$ is the mean of $\mathrm{x}, \sigma$ is the SD of $\mathrm{x}$, and $E(t)$ represents the expected value of the quantity $t$. Kurtosis is defined as $k=E(\mathrm{x}-\mu)^{4} / \sigma^{4}$, where $\mu$ is the mean of $\mathrm{x}, \sigma$ is the SD of $\mathrm{x}$, and $E(t)$ represents the expected value of the quantity $t$. The kurtosis of the normal distribution is 3. Other histogram features, including histogram mean, mode, full width at half maximum (FWHM), and SD, were recorded.

For segmentation, a 2-tissue compartment segmentation strategy was used, with FAST at default settings and partial volume segmentation. Segmentations were confirmed by histogram (Fig 1) as previously described. ${ }^{5}$

\section{Statistical Analysis}

For the brain volume growth curve, we first fitted the basic polynomial regression model, in which a third-degree polynomial of age was used to model the scaled brain volume growth curve. ${ }^{6}$ Sex and scanner identification (ID) covariables were then added, and the model was refitted. For characteristics of the CT density histogram, a polynomial regression of the second-degree of age (basic model) was fitted first, and the additional covariates such as sex and scanner ID were considered. Dependent variables were scaled by SD. Models were selected on the basis of the adjusted coefficient of determination $\left(R^{2}\right)$. Models were evaluated using leave-one-out crossvalidation (LOOCV), and root-mean-square error (RMSE) was computed for predictive accuracy. Curve fitting and statistical analysis were performed using GraphPad Prism software, Version 7.0c for Mac OS X (GraphPad Software, San Diego, California). The statistical significance of sex and scanner platform contribution were calculated in $\mathrm{R}$ statistical and computing software (http://www.r-project.org/). 

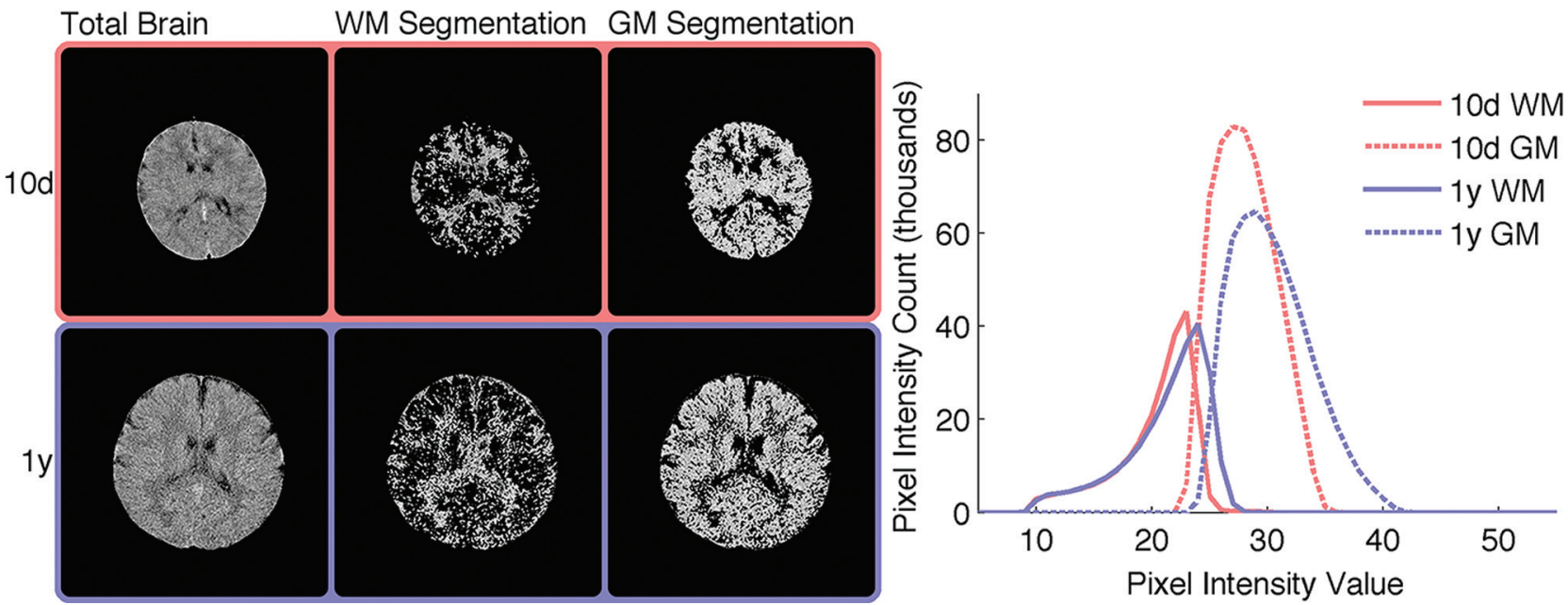

FIG 1. Brain-extracted head CT images with products of direct segmentation. Ten-day and 1-year-old brains were segmented. White matter segmentation is shown in the center panels, and gray matter segmentation is shown on the right. Histogram analysis of the products of segmentation shows a relative increase in the size of the gray matter peak and movement of the peak mode to a slightly higher density.
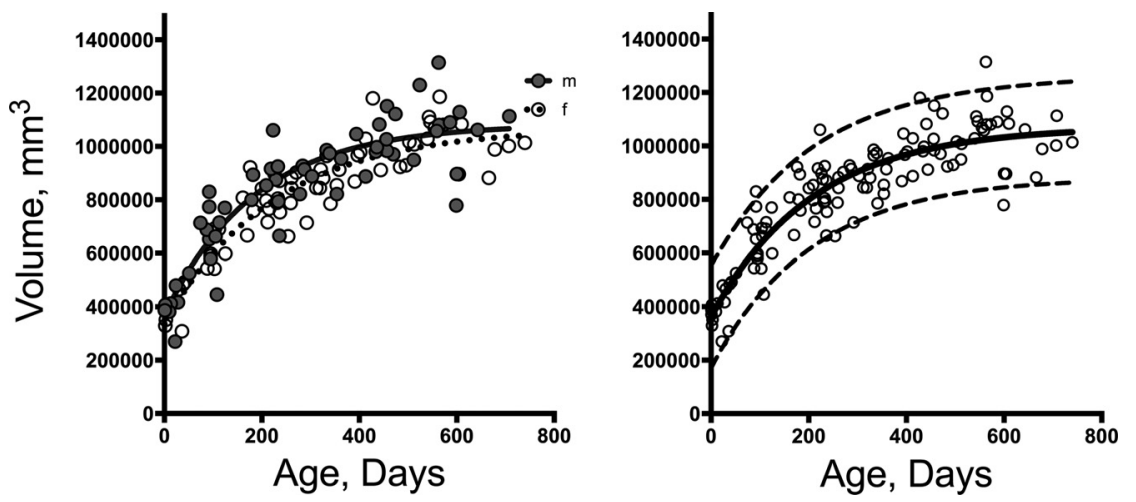

1)

$$
\begin{aligned}
& \mathrm{Vol} / 10,000= 7.97+20.63 \times \text { Age } \\
&-8.66 \\
& \times A g e^{2}+2.08 \times A g e^{3}+0.52 \\
& \times \operatorname{Sex}(\text { boy }) .
\end{aligned}
$$

At birth, the brain volume is between 300 and $400 \mathrm{~cm}^{3}$, greater than double in the first year of life (average volume at 1 year is $948 \mathrm{~cm}^{3}$ ), and growing an additional $19 \%$ in volume in the second year, to $1072 \mathrm{~cm}^{3}$. With a polynomial model with baseline volume at 0-30 days, brain volume increase is $150 \%$ in the first year (95\% CI, 144\%$150 \%)$ and $172 \%$ at year $2(95 \% \mathrm{CI}$, 148\%-197\%) (Fig 2, Table 1, and Equation 1). Regression results for Equation 1 indicated a $5200 \mathrm{~mm}^{3}$ significantly higher average brain volume for boys over girls $(P<.01)$, not adjusted for body weight.

Although adding both sex and scanner as covariates resulted in a smaller RMSE than adding sex only, the crossvalidation (LOOCV) RMSE indicated that adding both covariates resulted in overfitting the data (5.4\% versus $3.8 \%$ increase in RMSE, Table 1).

\section{RESULTS}

\section{Brain Volume Curve Modeling}

Brain volume as a function of age is shown in Fig 2, with trendlines and 95\% predictive bands. From Table 1, adjusted $R^{2}$ indicated that a third-degree polynomial of age with sex as a covariate performed the best in Equation 1, while adding the additional scanner did not improve the adjusted $R^{2}$.

\section{Brain Density Histogram Analysis}

The radiodensity of the brain changes as a function of age (Fig 3), and the changes can be quantified through analysis of the radiodensity histogram metrics (Fig 4, trendlines and 95\% predictive bands are shown). Model-fitting results indicated that a sec- 
ond-degree polynomial of age with an additional scanner ID performed the best for mean, mode, and kurtosis; first-degree of age with scanner ID performed best for FWHM; and $\sigma$, firstdegree of log (age) with scanner ID performed best for skewness (Equation 2). Cross-validation (LOOCV) results on scaled dependent variables indicated that our models had good predictive power in CT histogram data because the RMSE from LOOCV demonstrated small differences $(3 \%-7 \%)$ from the RMSE from the polynomial model (Table 2).
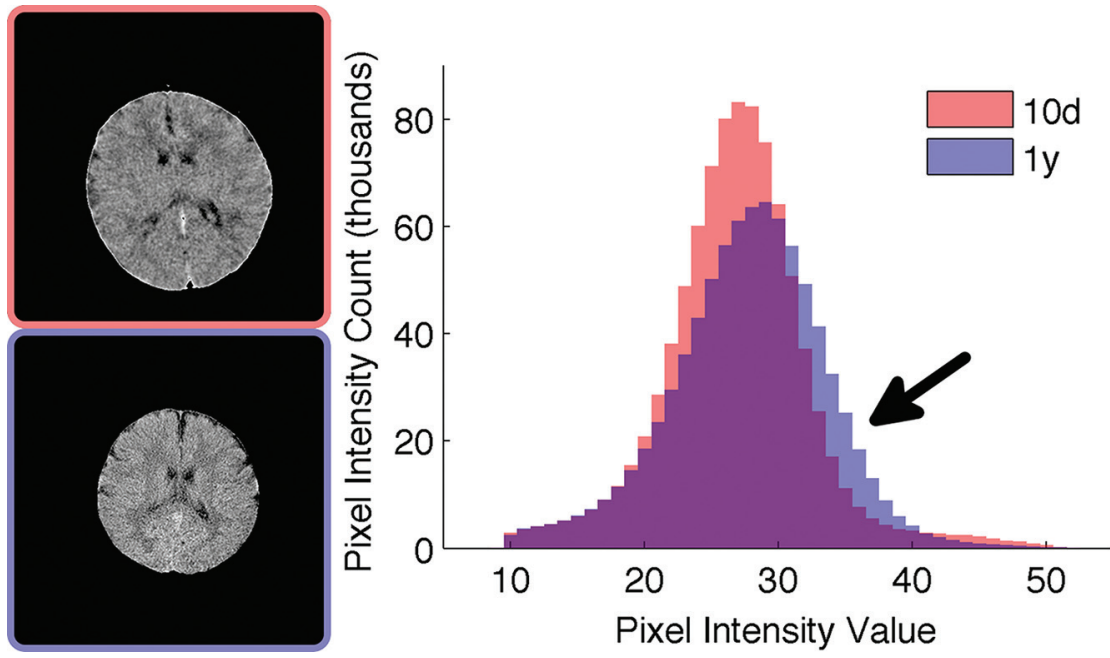

FIG 3. Histogram analysis of brain-extracted head CT images, at 10 days and 1 year. The histogram becomes more symmetric with age, and the leading edge at 1 year (arrow) reflects higher density voxels. $y$ indicates year; $d$, day.
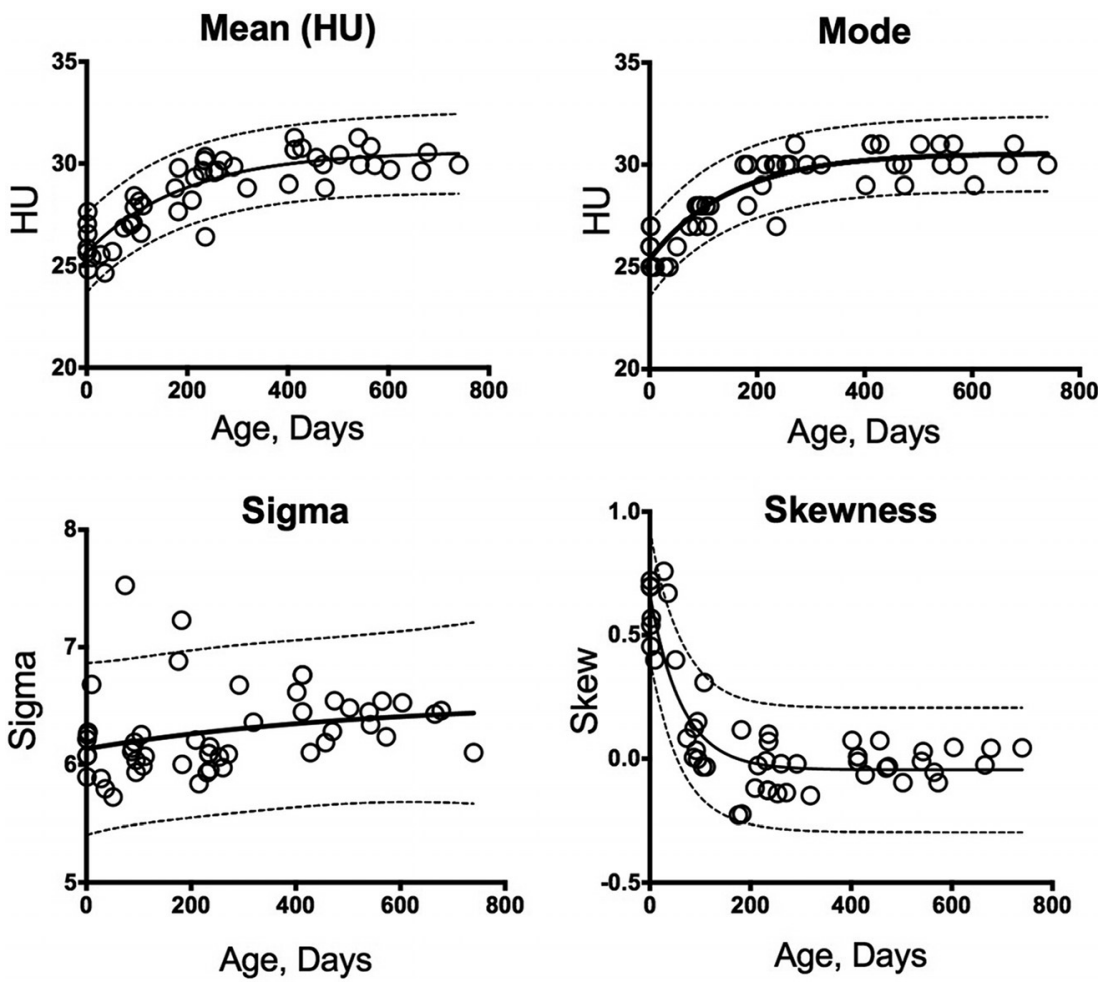

$$
\times A g e+\beta_{2} \times A g e^{2} .
$$

Histogram mean and modal Hounsfield density curves parallel the brain volume changes that occur in the first 500 days of life (Fig 4). Mean brain tissue density increases from $25.6 \mathrm{HU}$ to 30.6 HU during this period (Tables 2). The skewness of the brain tissue density histogram decreases logarithmically from the time of birth to a zero value at approximately 200 days. The total brain Hounsfield unit histogram kurtosis also decreases during this period to approach a value of 3 , representing a normal distribution. With concern that differences in scanner acquisition and calibration may impact the characteristics of the radiodensity histogram, data from the different scan platforms were analyzed separately. Histogram analysis showed similar trends from all 3 scan platforms (On-line Figs 1 and 2). A composite model inclusive of all 3 scan platforms was also derived (On-line Appendix and On-line Table).

\section{Gray and White Matter Segmentation}

Direct segmentation of images from a single scan platform (scanner 2) permits an analysis of gray and white matter changes as a function of brain develop-
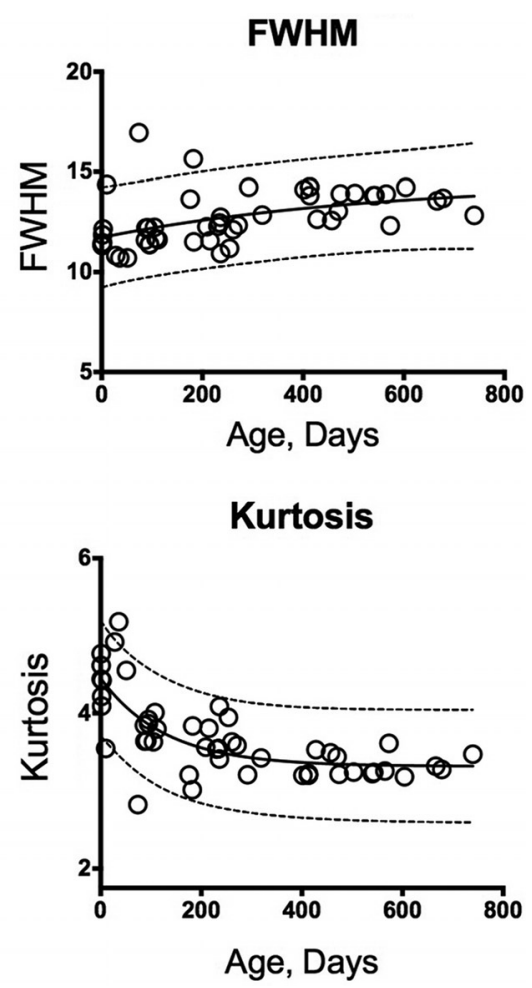

FIG 4. Total brain histogram analysis from 1 scan platform (scanner 2) shows total brain Hounsfield unit mean and Hounsfield unit histogram mode as well as FWHM, histogram SD $(\sigma)$, histogram skewness, and kurtosis as a function of subject age. Trendline and $95 \%$ predictive bands are shown. 
Table 2: Model fitting and cross-validation results for scaled CT radiodensity histogram (scanner 2)

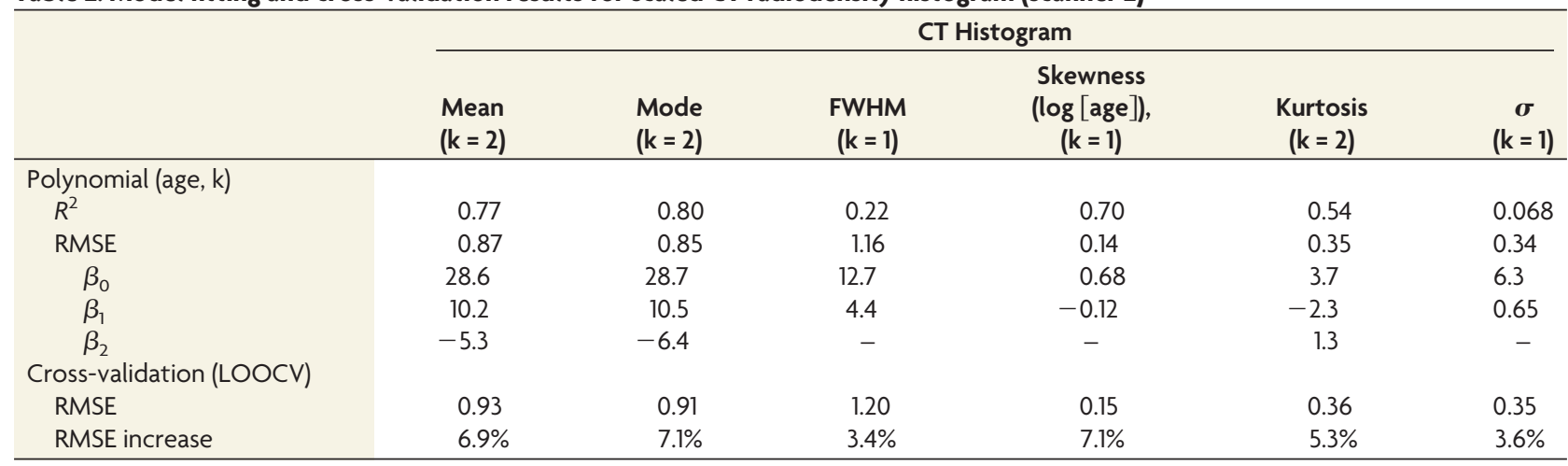

Note:- $\mathrm{k}$ indicates order/degree of the polynomial.

Gray and White Matter Volume

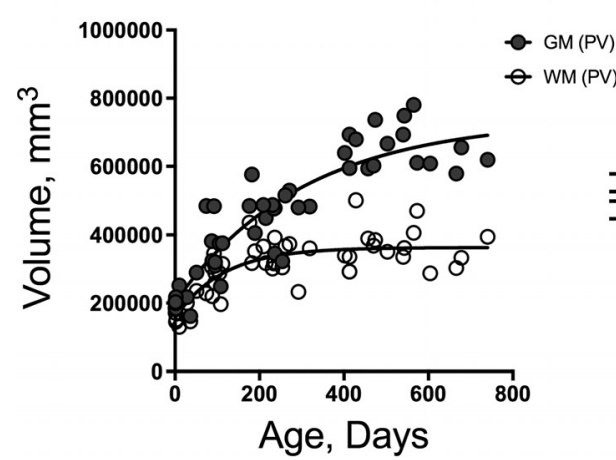

Gray and White Matter Density

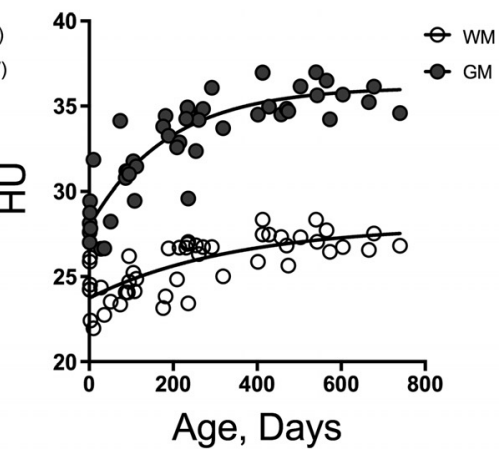

Normalized Gray and White Matter Volume

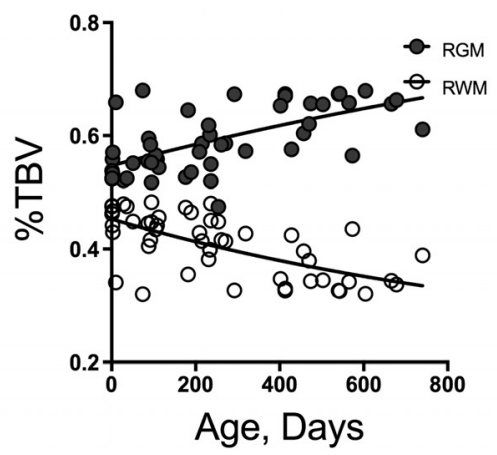

FIG 5. Direct segmentation into gray and white matter with nonlinear regression as a function of age. Tissue volumes (left), tissue densities (center), and tissue volumes normalized to total brain volume (right) are shown. PV indicates partial volume; RGM, relative gray matter volume; RWM, relative white matter volume.

ment (Figs 1 and 5). Both gray and white matter volumes increase in the perinatal period, with the greatest changes occurring in the first 200 days of life (Fig 5). Gray and white matter volumes appear nearly convergent at birth, at approximately $200 \mathrm{~cm}^{3}$. Gray matter volume increases dramatically in the first year, to approximately $500 \mathrm{~cm}^{3}$, a $150 \%$ increase, whereas white matter increases to approximately $350 \mathrm{~cm}^{3}$, a $75 \%$ increase. Segmented tissue volumes normalized to total brain volumes show a relative increase in gray matter volume from $55 \%$ of brain volume to $65 \%$ at 1 year, while the relative white matter volume decreases proportionally in the same period. Concurrent with these changes, both gray and white matter densities increase, with gray matter from $28 \mathrm{HU}$ at birth to $36 \mathrm{HU}$ at 1 year and WM from $23.7 \mathrm{HU}$ at birth to $26 \mathrm{HU}$ at 1 year.

\section{DISCUSSION}

Although several studies of quantitative imaging of brain growth and development have focused on the immediate perinatal period, ${ }^{8-10}$ few studies have focused on the first years of life. ${ }^{11-13}$ This time window is important in brain development because the largest changes in brain maturation occur in the first 2 years. ${ }^{14,15}$ Subtle abnormalities of brain size and abnormal gray and white matter volumes and growth trajectories have been associated with diseases such as attention deficit/hyperactivity disorder, ${ }^{6,16}$ autism, ${ }^{17,18}$ and schizophrenia. ${ }^{19}$ Reference data in this time window are also needed because neonates and toddlers can pose a challenge for diagnostic medicine in the clinical setting, where history and physical examination may be limited. Most in vivo studies are based on MR imaging data, with the few studies based on CT imaging having been performed before the wide use of MR imaging. ${ }^{10,13,20}$ To our knowledge, this is the first study to investigate the changes in brain development by digital analysis of head CT data. The logistics of MR imaging of toddlers can limit investigation into brain development in this period of childhood, and additional data derived from head CT images serve to support and augment data obtained from MR imaging studies.

Our CT-derived brain volumes are overall consistent with reported MR imaging findings, though no true curve fitting has yet been reported for brain growth in this period of brain development, to our knowledge. In an early MR imaging study with emphasis on brain development of premature and mature neonates, Hüppi et $\mathrm{al}^{9}$ found a near-linear growth curve in the immediate perinatal period and a birth volume at maturity of approximately $400,000 \mathrm{~mm}^{3}$ at full-term. Also using MR imaging, Holland et al ${ }^{8}$ investigated brain growth trajectories and rates of change in the first 3 months of infant brain development, finding a near-linear growth curve in the first 90 days of life and a birth volume of $347,388 \mathrm{~mm}^{3}$, similar to our finding of $361,494 \mathrm{~mm}^{3}$. Choe et $\mathrm{al}^{11}$ studied infant brain development from 3 to 13 months, recruiting 27 full-term and developing neonates, some scanned more than once, with scans at 3, 6, and 12 months. Although the emphasis of that study was on relative growth of segmented brain structures, 
the study recorded total brain volumes at approximately $650 \mathrm{~cm}^{3}$ at 16 weeks, $800 \mathrm{~cm}^{3}$ at 30 weeks, and $950 \mathrm{~cm}^{3}$ at 52 weeks, similar to our findings, and with individual variation (data scatter) similar to the variation that we report. ${ }^{11}$

Studies by Knickmeyer et $\mathrm{al}^{12}$, and Shi et $\mathrm{al}^{21}$ are of similar design and offer the most complete MR imaging data regarding brain growth during the first 2 years of life, with cohorts scanned in the neonatal period (at 2-4 weeks) and at 1 and 2 years. This study design with clustering of the data at discrete time points does not permit generation of a detailed growth curve but does show comparable brain volumes with approximately $400 \mathrm{~cm}^{3}$ at 2-4 weeks and $800-900 \mathrm{~cm}^{3}$ at 12 months, with a plateau at approximately $1000 \mathrm{~cm}^{3}$ at 24 months seen in both studies, with a qualitative growth curve and intersubject variation (data scatter) similar to those in the current study.

Previous studies have investigated sex differences in perinatal brain volume with mixed conclusions, likely due to differences in the ages of the children being evaluated and small sample sizes. ${ }^{8,12}$ Similar to the prospective MR imaging findings of Shi et al, ${ }^{21}$ we found a small but statistically significant difference in brain growth curves between boys and girls, with boys being consistently larger, approximately 5.5\% larger at 1 year and 5\% larger at 2 years. These numbers were not normalized to body size. Statistical analysis also revealed that the use of different scan platforms does not contribute significantly to the volume estimate or the fitted growth curve.

Our study investigated the correlation between the parametric measures of the brain density histogram and subject age. Although whole-brain histogram analysis has been explored in a number of different MR imaging techniques, histogram analysis of head CT images has not been widely investigated, to our knowledge. Histogram analysis offers objective, quantitative evaluation of parameters beyond those seen at routine diagnostic image interpretation and lends itself to automated analysis of head CT imaging. The clear age-dependent trends of the data motivated the concept of a statistical model. Our retrospective study design resulted in a continuous age distribution, which enabled curve fitting, which is not possible with prospective studies with discrete age cohorts. ${ }^{11,16}$

Total brain density histogram analysis as a function of age yields several findings. The overall brain tissue density (mean density) increases in the neonatal period, consistent with early reports using single-slice brain CT ROI methods. ${ }^{13}$ The shape of the density histogram also changes, with rapidly decreasing histogram skewness in the first 200 days of life, reflecting a shift of the histogram toward higher density and an increasing percentage of higher density voxels during this period (Fig 3). Major changes in brain tissue known to occur in this time include an overall decrease in the percentage of water in the tissue, ${ }^{22}$ and a relative increase in gray matter volume. ${ }^{9,12,23}$ Both factors would be expected to contribute to the increase in density and the changes in the attenuation histogram.

Direct segmentation of brain CT yields gray and white matter maps, which can be characterized for density and volume, showing a relative increase in gray matter in the first 2 years, with steeper changes in the first 200 days, as well as an increase in both gray and white matter density during early brain development.
Previous studies based on MR imaging have shown that rapid changes in brain volume occurring in the immediate postnatal period relate to an increase in gray matter volume, ${ }^{9,12,23,24}$ which, in turn, is thought to reflect synapse formation and the development of the neuropil. ${ }^{12}$ Decreases in water diffusion seen in brain tissue during this time may reflect, in part, myelination and a decreasing tissue-water fraction. ${ }^{15,22}$

Although clinical CT machines are subject to routine calibration, with an accepted margin of error, ${ }^{25}$ the effect of variations between machines becomes important in quantitative studies. Our curve fitting represents a feasibility study rather than a true predictive, universal, or optimal model, however, because various alternative statistical models were not evaluated and the contributions of small differences in scan platform and data acquisition were not fully explored. Rather our goal was to illustrate the feasibility of such model development based on digital analysis of head CT images. Appreciation of the possibility of such modeling serves to emphasize the value of quantitative imaging and underscore the value of higher standards in image acquisition and hardware calibration.

\section{CONCLUSIONS}

Changes in brain radiodensity are highly correlated with developmental age, and normative metrics of the brain radiodensity histogram derived from routine clinical head CT images can be used to develop a model of normal brain development. Histogram analysis is amenable to automation, and digital analysis together with normative metrics derived from head CT images may be used to improve the sensitivity of head CT in the detection of subtle brain injury and neurodevelopmental disorders.

\section{REFERENCES}

1. CT Accreditation Program: Testing Instructions. Revised January 6, 2017. https://www.acraccreditation.org/ /media/ACRAccreditation/ Documents/CT/CT-Accreditation-Testing-Instructions.pdf, Accessed August 15, 2017

2. Zhang Y, Brady M, Smith S. Segmentation of brain MR images through a hidden Markov random field model and the expectationmaximization algorithm. IEEE Trans Med Imaging 2001;20:45-57 CrossRef Medline

3. Jenkinson M, Beckmann CF, Behrens TE, et al. FSL. Neuroimage 2012;62:782-90 CrossRef Medline

4. Muschelli J, Ullman NL, Mould WA, et al. Validated automatic brain extraction of head CT images. Neuroimage 2015;114:379-85 CrossRef Medline

5. Cauley KA, Och J, Yorks PJ, et al. Automated segmentation of head computed tomography images using FSL. J Comput Assist Tomogr 2018;42:104-10 CrossRef Medline

6. Castellanos FX, Lee PP, Sharp W, et al. Developmental trajectories of brain volume abnormalities in children and adolescents with attention-deficit/hyperactivity disorder. JAMA 2002;288:1740-48 CrossRef Medline

7. R Core Team. R: A Language and Environment for Statistical Computing. Vienna, Austria: R Foundation for Statistical Computing; 2014. http://www.R-project.org/. Accessed January 3, 2015

8. Holland D, Chang L, Ernst TM, et al. Structural growth trajectories and rates of change in the first 3 months of infant brain development. JAMA Neurol 2014;71:1266-74 CrossRef Medline

9. Hüppi PS, Warfield S, Kikinis R, et al. Quantitative magnetic resonance imaging of brain development in premature and mature newborns. Ann Neurol 1998;43:224-35 CrossRef Medline

10. Brant-Zawadzki M, Enzmann DR. Using computed tomography 
of the brain to correlate low white-matter attenuation with early gestational age in neonates. Radiology 1981;139:105-08 CrossRef Medline

11. Choe MS, Ortiz-Mantilla S, Makris N, et al. Regional infant brain development: an MRI-based morphometric analysis in 3 to 13 month olds. Cereb Cortex 2013;23:2100-17 CrossRef Medline

12. Knickmeyer RC, Gouttard S, Kang C, et al. A structural MRI study of human brain development from birth to 2 years. J Neurosci 2008;28: 12176-82 CrossRef Medline

13. Penn RD, Trinko B, Baldwin L. Brain maturation followed by computed tomography. J Comput Assist Tomogr 1980;4:614-16 CrossRef Medline

14. Pfefferbaum A, Mathalon DH, Sullivan EV, et al. A quantitative magnetic resonance imaging study of changes in brain morphology from infancy to late adulthood. Arch Neurol 1994;51:874-87 CrossRef Medline

15. Zhang L, Thomas KM, Davidson MC, et al. MR quantitation of volume and diffusion changes in the developing brain. AJNR Am JNeuroradiol 2005;26:45-49 Medline

16. Proal E, Reiss PT, Klein RG, et al. Brain gray matter deficits at 33year follow-up in adults with attention-deficit/hyperactivity disorder established in childhood. Arch Gen Psychiatry 2011;68:1122-34 CrossRef Medline

17. Petropoulos H, Friedman SD, Shaw DW, et al. Gray matter abnor- malities in autism spectrum disorder revealed by $\mathrm{T} 2$ relaxation. Neurology 2006;67:632-36 CrossRef Medline

18. Courchesne E, Karns CM, Davis HR, et al. Unusual brain growth patterns in early life in patients with autistic disorder: an MRI study. Neurology 2001;57:245-54 CrossRef Medline

19. Lei W, Deng W, Li M, et al. Gray matter volume alterations in firstepisode drug-naive patients with deficit and nondeficit schizophrenia. Psychiatry Res 2015;234:219-26 CrossRef Medline

20. Hamano K, Iwasaki N, Kawashima K, et al. Volumetric quantification of brain volume in children using sequential CT scans. Neuroradiology 1990;32:300-03 CrossRef Medline

21. Shi F, Yap PT, Wu G, et al. Infant brain atlases from neonates to 1and 2-year-olds. PLoS One 2011;6:e18746 CrossRef Medline

22. Dobbing J, Sands J. Quantitative growth and development of human brain. Arch Dis Child 1973;48:757-67 CrossRef Medline

23. Gilmore JH, Lin W, Prastawa MW, et al. Regional gray matter growth, sexual dimorphism, and cerebral asymmetry in the neonatal brain. J Neurosci 2007;27:1255-60 CrossRef Medline

24. Matsuzawa J, Matsui M, Konishi T, et al. Age-related volumetric changes of brain gray and white matter in healthy infants and children. Cereb Cortex 2001;11:335-42 CrossRef Medline

25. Allen JS, Damasio H, Grabowski TJ. Normal neuroanatomical variation in the human brain: an MRI-volumetric study. Am J Phys Anthropol 2002;118:341-58 CrossRef Medline 